\title{
Recent Results and Perspectives for Precision Astrometry and Photometry with Adaptive Optics.
}

\author{
Jessica R. Lu ${ }^{\mathrm{a}}$, Andrea M. Ghez ${ }^{\mathrm{b}, \mathrm{c}}$, Sylvana Yelda ${ }^{\mathrm{b}}$, Tuan Do ${ }^{\mathrm{b}}$, Will Clarkson ${ }^{\mathrm{b}}$, Nate \\ McCrady ${ }^{\mathrm{d}}$, Mark Morris ${ }^{\mathrm{b}}$ \\ ${ }^{a}$ California Institute of Technology, MC 249-17, Pasadena, CA 91125; \\ ${ }^{b}$ UCLA Department of Physics and Astronomy, Los Angeles, CA 90095; \\ ${ }^{c}$ UCLA Institute of Geophysics and Planetary Physics, Los Angeles, CA 90095; \\ dUniversity of Montana, Missoula, MT 59812;
}

\begin{abstract}
Large ground-based telescopes equipped with adaptive optics (AO) systems have ushered in a new era of highresolution infrared photometry and astrometry. Relative astrometric accuracies of $<0.2$ mas have already been demonstrated from infrared images with spatial resolutions of 55-95 mas resolution over 10-20" fields of view. Relative photometric accuracies of 3\% and absolute photometric accuracies of 5\%-20\% are also possible. I will review improvements and current limitations in astrometry and photometry with adaptive optics of crowded stellar fields. These capabilities enable experiments such as measuring orbits for brown dwarfs and exoplanets, studying our Galaxy's supermassive black hole and its environment, and identifying individual stars in young star clusters, which can be used test the universality of the initial mass function.
\end{abstract}

Keywords: adaptive optics, astrometry

\section{INTRODUCTION}

Scientific results from adaptive optics $(\mathrm{AO})$ systems on ground-based telescopes have increased dramatically over the last $\sim 10$ years. As existing AO systems have matured and stabilized and the number of scientifically productive $\mathrm{AO}$ systems has increased, AO observations have become more routine. With this stability, we are now exploring the most effective ways to extract high-precision quantitative science from AO observations. In this proceeding, we review the current state of astrometric $(\S 2)$ and photometric ( $\$ 3$ and $\S 4$ ) accuracies achieved with existing AO systems. We also highlight the limitations of current measurements in an effort to determine where improvements can be made. Each section begins with a list of several science results utilizing AO astrometric or photometric measurements and the accuracies achieved, followed by a discussion of the limiting factors. Precision AO astrometry, in particular, is behind some of the driving science cases for future AO systems and large telescopes (e.g. TMT, E-ELT). Understanding the current and future limitations of AO astrometric and photometric measurements is still a work in progress.

\section{RELATIVE ASTROMETRY}

The increased spatial resolution provided by adaptive optics observations in the near-infrared not only enables observations of crowded fields or close binary stars, but also provides improved astrometric precision. AO astrometry has been used to measure dynamical masses of brown dwarfs, ${ }^{1,2}$ study our Galaxy's central supermassive black hole and its environment, ${ }^{3-6}$ and study the formation and evolution of young star clusters ${ }^{7}$ and massive stars. $^{8}$

Astrometric accuracies of $<0.2$ mas have been demonstrated with the Keck and Palomar AO systems over multiple nights, months, and even years of observing. ${ }^{9-11}$ These accuracies are best determined by measuring the residuals from linear motion for many stars within a crowded-field, such as the Galactic center (see Figure 1 , blue line).

Send correspondence to jlu@astro.caltech.edu

Adaptive Optics Systems II, edited by Brent L. Ellerbroek, Michael Hart, Norbert Hubin, Peter L. Wizinowich, Proc. of SPIE Vol. 7736, 77361I · @ 2010 SPIE · CCC code: 0277-786X/10/\$18 · doi: 10.1117/12.859737 


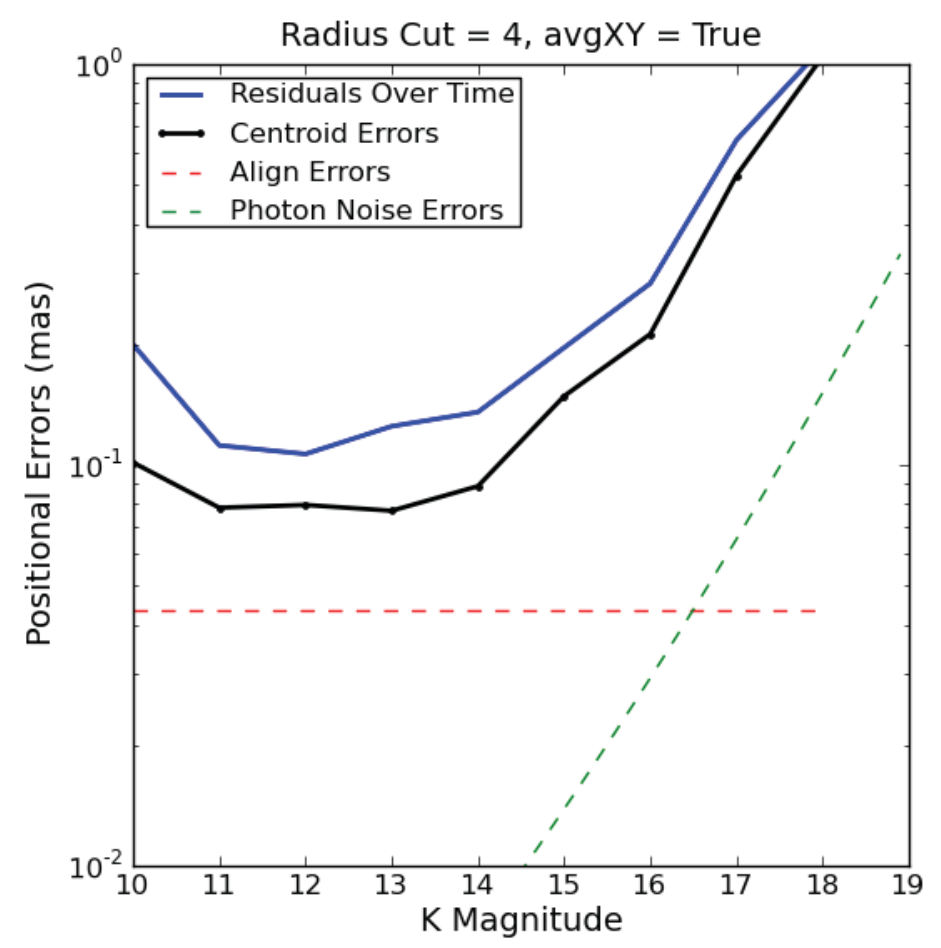

Figure 1. Astrometric error as a function of stellar brightness for Keck/NIRC2 LGS AO observations of stars in the Galactic center. The average random error for each data set, hereafter referred to as the "centroiding error" (black solid), is determined by running the Starfinder PSF fitting routine on 3 subsets of the data and calculating the error on the mean position for each star. Each star's motion over time is fit with a linear motion model and the residuals from these fits give the astrometric accuracy (blue solid). Errors due to aligning data sets are negligible (red dashed). Errors due to photon noise are calculated by simulating star fields with input positions and fluxes and a known PSF then comparing to the fluxes recovered by Starfinder (green dashed).

Astrometric observations from ground-based telescopes are fundamentally limited by differential tip-tilt jitter, which, fortunately, reduces with integration time as $1 / \sqrt{t_{\text {int }}} .{ }^{12}$ This behavior has been verified on sky by plotting the astrometric precision as a function of total integration time for AO observations taken within a single night (Figure 2). ${ }^{9,10}$ Practically, astrometric measurements are more often limited by systematic effects due to residual optical distortions, differential atmospheric refraction, ${ }^{13}$ and PSF errors. Both optical distortions from the camera and differential atmospheric refraction (DAR) can be calibrated and corrected. For instance, Yelda et al. (in preparation) have used on-sky observations of globular clusters to characterize the Keck NIRC2 distortion solution to the 1 mas level, a factor of 3 improvement over previous solutions using pinhole masks. The relative distortion error felt between epochs is also sensitive to the observational design. To achieve the highest astrometric precision, residual distortions are minimized in Keck/UCLA Galactic center astrometric measurements by always observing with small dithers* and at the same detector orientation and position. ${ }^{3}$ Without this careful observing planning, residual distortions may be the dominant source of astrometric error for bright, uncrowded sources (see Figure 14 in Fritz et al. ${ }^{14}$ ). Uncorrected differential atmospheric refraction can also contribute 1-3 mas of error for two stars separated by 5"; however, it can be modeled and images corrected using real-time weather data and a simple model of the Earth's atmosphere.

The limiting factor for crowded-field AO astrometry appears to be PSF errors, as shown by Fritz et al., ${ }^{14}$ even when there are plenty of stars to derive an empirical PSF using codes such as Starfinder. ${ }^{15}$ Figure 3 shows

${ }^{*}$ We still dither in order to remove the effects of bad pixels, reduce the impact of intra-pixel sensitivity variations, and average over small-scale distortions that can't easily be characterized. 


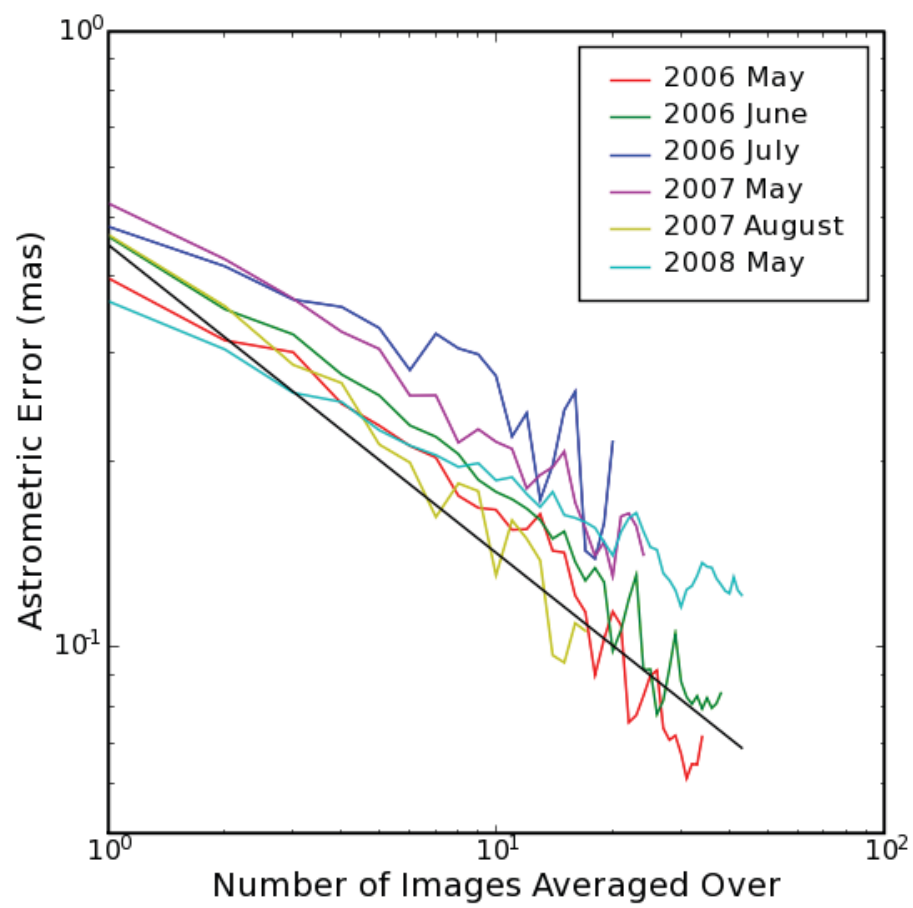

Figure 2. Reproduced from Lu, ${ }^{10}$ figure 4.56. Astrometric error vs. integration time for several Keck LGS AO data sets of the Galactic center. The data closely follow the expected trend of $1 / \sqrt{t_{\text {int }}}$ shown by the solid black line.

astrometric errors vs. stellar brightness resulting from PSF errors for VLT NAOS/CONICA observations of the Galactic center as described by Fritz et al. ${ }^{14}$ At the bright end, their astrometry is limited by residual distortion (see Figure 14 in Fritz et al. ${ }^{14}$ ) since their data sets are widely dithered. However, after this, the dominant source of astrometric error at the bright end is from errors in the PSF fitting process when using the wrong PSF to extract a star, a brightness-independent process. At the faint end, astrometry is also limited by errors in the PSF (see Equation 10 in Fritz et al. ${ }^{14}$ ). This source of error is a result of incorrectly subtracted PSFs from brighter neighboring stars resulting in residuals that bias the astrometry of fainter sources; hence the error depends on stellar brightness. These PSF errors depend on the Strehl of the observations, the amount of PSF variability over the field of view, and the exact crowding of the science field being observed, so these numbers are not exactly applicable to other observations. However, PSF errors are still likely to be a significant source of error for most crowded-field astrometric experiments. In summary, calculations by Clarkson et al. (in preparation) suggest that the total unaccounted for error between the accuracies calculated from stellar motion residuals and the centroiding errors is $\sim 0.15$ mas for bright sources in Keck NIRC2 observations of the crowded Galactic center Arches cluster at several epochs. When combined with the centroiding error within each epoch, this results in an astrometric accuracy of 0.2 mas.

\section{RELATIVE PHOTOMETRY}

Relative photometry from adaptive optics observations has been used to study both the time variability of individual sources and the flux ratios or luminosity functions of multiple systems and clusters of stars. For instance, AO observations of Sgr A*-IR, the accreting supermassive black hole at the Galactic center, have been used to study its time variability within a single night ${ }^{16-18}$ ). Also, AO observations have been used to construct luminosity functions in massive young star clusters, which can be converted into initial mass functions in order to probe how star formation may differ in the most crowded star clusters. ${ }^{19}$ 


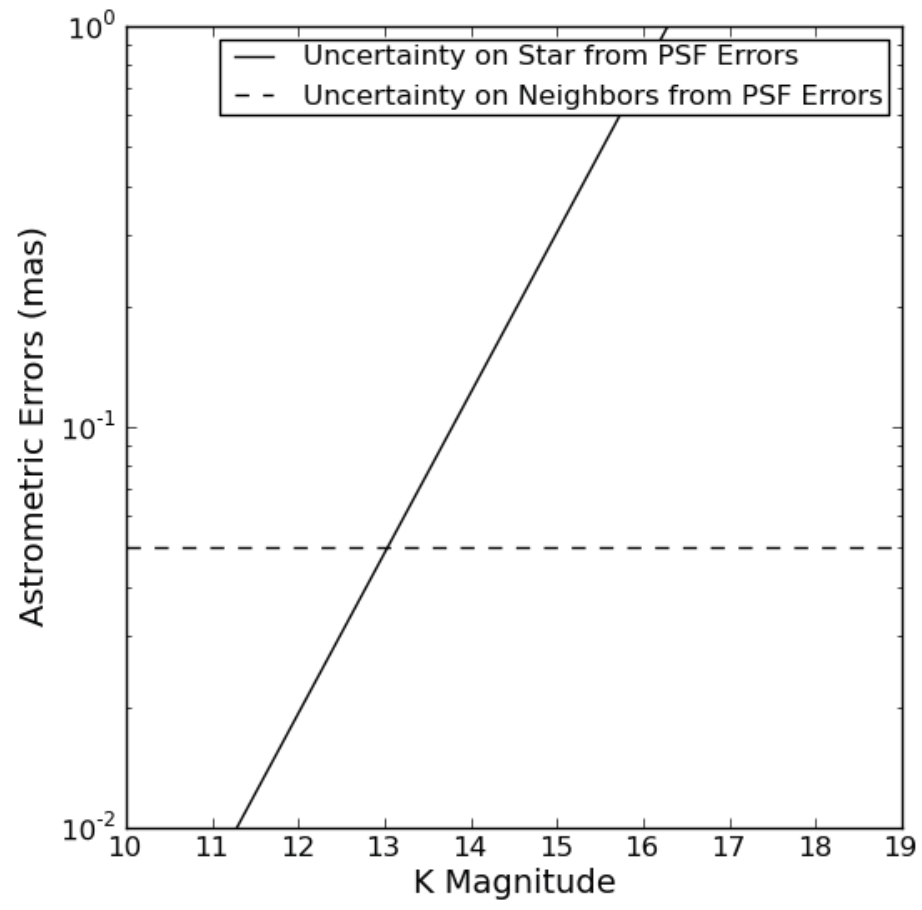

Figure 3. Astrometric errors vs. stellar brightness resulting from PSF errors for VLT NAOS/CONICA observations of the Galactic center. ${ }^{14}$ At the bright end, their astrometry is limited by residual distortion (see Figure 14 in Fritz et al. ${ }^{14}$ ) since their data sets are widely dithered. However, after this, the dominant source of astrometric error at the bright end is from errors in the PSF fitting process when using the wrong PSF to extract a star, a brightness-independent process. At the faint end, astrometry is also limited by errors in the PSF (see Equation 10 in Fritz et al. ${ }^{14}$ ). This source of error is a result of incorrectly subtracted PSFs from brighter neighboring stars resulting in residuals that bias the astrometry of fainter sources; hence the error depends on stellar brightness.

Relative photometric accuracies of 3-4\% have been demonstrated with AO observations. These accuracies can be reached within a single night of $\mathrm{AO}$ observations, under the same atmospheric and $\mathrm{AO}$ conditions, as has been demonstrated ${ }^{20,21}$ from RMS photometric errors for individual stars across many exposures throughout a night. Additionally, by comparing photometry for crowded star fields such as the Galactic center across many nights taken over several years, we show in Figure 4 that the RMS photometric errors of $4 \%$ are still reached. Current limitations on relative photometry are due to errors in the PSF estimates in these crowded fields. Analysis by Schoedel et al. ${ }^{21}$ shows that extracting the PSF from images using different PSF stars fully accounts for the relative photometric errors and is orders of magnitude above the photon noise limit for bright stars (also see Esslinger \& Edmunds ${ }^{22}$ ).

\section{ABSOLUTE PHOTOMETRY}

Absolute photometry is necessary for multi-wavelength analysis such as for studies of stellar populations. ${ }^{19,23}$ Typically, AO observations are absolutely photometrically calibrated by using existing seeing-limited or HST observations taken in different filters and with very different resolutions, requiring filter conversions and crowding analysis. Absolute photometry of new fields typically requires observations of standard stars over a range of airmasses on a photometric night. We have attempted such a program using the Keck AO system and the NIRC2 instrument, observing 3 standard stars in natural guide star (NGS) mode at airmasses of 1.0-1.8 in H, K', and L' filters. Using standard IRAF aperture photometry reduction packages and a 2" aperture radius, we measure the zeropoints for these three filters with uncertainties of $0.02 \mathrm{mag}, 0.02 \mathrm{mag}$, and $0.06 \mathrm{mag}$ at 


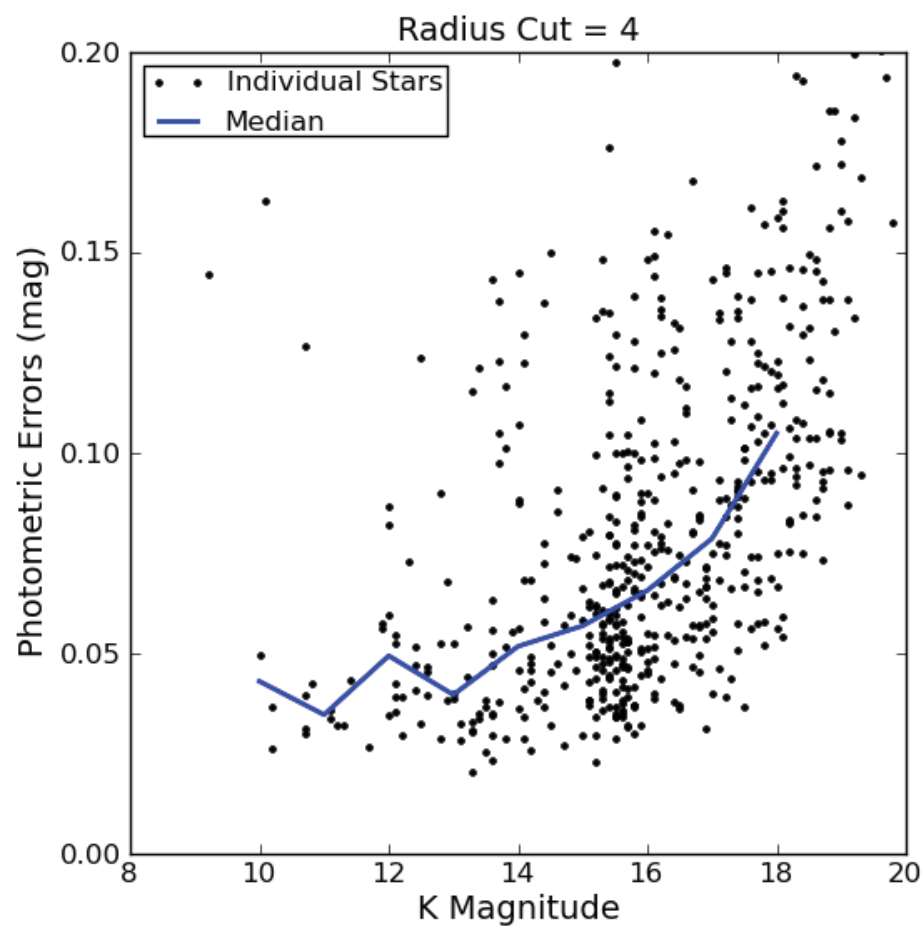

Figure 4. Relative photometric errors for AO observations of the Galactic center at K'-band as a function of stellar brightness. The photometric errors are calculated as the RMS error of 11 individual photometric measurements taken over several years. For bright stars, the median photometric accuracy is $\sim 4 \%$. No cuts have been made to remove intrinsically variable sources, which may contribute to the large spread in photometric errors. The individual data sets were photometrically calibrated using 10 non-variable stars. The data sets are taken with Keck/NIRC2 LGS AO and are described in detail in Yelda et al. (in preparation) and references therein.

H, K', and L', respectively. However, applying these zeropoints to crowded-field LGS AO observations, where the size of the empirically estimated PSF is only $\sim 0^{\prime \prime} .5-1^{\prime \prime} .0$, depends on proper aperture corrections between the science-field PSF and the photometric standard star's aperture. The variable AO correction between the NGS observations of the bright standard stars and the LGS observations, with faint off-axis tip-tilt stars, of a Galactic star cluster, for instance, result in different PSFs that cannot easily be matched at radii of $<0^{\prime \prime} .5$. Sheehy et al. ${ }^{24}$ proposed to model the PSF from the science observations directly out to large radii by assuming the 1D power spectrum of the image can be described entirely from the distribution of stars, a noise floor, and a PSF constructed from the telescope, camera detector, and AO-corrected atmosphere. With this technique they achieve $4 \%$ photometric accuracy across multiple exposures of the same field. However, when compared with HST observations of the same field they find an RMS error of only $20 \%$, some of which may be attributed to intrinsic source variability. Again, improved knowledge of the complete PSF for crowded stellar fields would provide better aperture corrections permitting absolute photometric accuracies of $<5 \%$ for fields photometrically calibrated only from AO observations, rather than bootstrapping from lower spatial resolution observations from 2MASS, UKIDSS, or HST.

\section{CONCLUSIONS}

Astrometric and photometric measurements with adaptive optics observations have achieved accuracies of

- Relative Astrometric Errors: $<0.2$ mas 
- Relative Photometric Errors: 0.03 - $0.04 \mathrm{mag}$

- Absolute Photometric Errors: $<0.05$ mag (however, see discussion in $\S 4$ ).

The current limitations of such measurements are primarily due to uncertainties in the PSF. Efforts in PSFreconstruction from AO telemetry, ${ }^{25}$ modeling the spatial dependence of the PSF from $\mathrm{Cn}^{2}$ profiles ${ }^{26}$ and better methods for fitting the PSF empirically for crowded fields will be needed to improve upon the above accuracies.

\section{REFERENCES}

[1] Dupuy, T. J., Liu, M. C., and Ireland, M. J., "Dynamical Mass of the Substellar Benchmark Binary HD 130948BC," ApJ 692, 729-752 (Feb. 2009).

[2] Konopacky, Q. M., Ghez, A. M., Barman, T. S., Rice, E. L., Bailey, J. I., White, R. J., McLean, I. S., and Duchêne, G., "High-precision Dynamical Masses of Very Low Mass Binaries," ApJ 711, 1087-1122 (Mar. 2010).

[3] Ghez, A. M., Salim, S., Weinberg, N. N., Lu, J. R., Do, T., Dunn, J. K., Matthews, K., Morris, M. R., Yelda, S., Becklin, E. E., Kremenek, T., Milosavljevic, M., and Naiman, J., "Measuring Distance and Properties of the Milky Way's Central Supermassive Black Hole with Stellar Orbits," ApJ 689, 1044-1062 (Dec. 2008).

[4] Gillessen, S., Eisenhauer, F., Trippe, S., Alexander, T., Genzel, R., Martins, F., and Ott, T., "Monitoring Stellar Orbits Around the Massive Black Hole in the Galactic Center," ApJ 692, 1075-1109 (Feb. 2009).

[5] Lu, J. R., Ghez, A. M., Hornstein, S. D., Morris, M. R., Becklin, E. E., and Matthews, K., "A Disk of Young Stars at the Galactic Center as Determined by Individual Stellar Orbits," ApJ 690, 1463-1487 (Jan. 2009).

[6] Bartko, H., Martins, F., Fritz, T. K., Genzel, R., Levin, Y., Perets, H. B., Paumard, T., Nayakshin, S., Gerhard, O., Alexander, T., Dodds-Eden, K., Eisenhauer, F., Gillessen, S., Mascetti, L., Ott, T., Perrin, G., Pfuhl, O., Reid, M. J., Rouan, D., Sternberg, A., and Trippe, S., "Evidence for Warped Disks of Young Stars in the Galactic Center," ApJ 697, 1741-1763 (June 2009).

[7] Stolte, A., Ghez, A. M., Morris, M., Lu, J. R., Brandner, W., and Matthews, K., "The Proper Motion of the Arches Cluster with Keck Laser-Guide Star Adaptive Optics," ApJ 675, 1278-1292 (Mar. 2008).

[8] Cameron, P. B., The Formation and Evolution of Neutron Stars: Astrometry, Timing, and Transients, PhD thesis, Caltech (June 2008).

[9] Cameron, P. B., Britton, M. C., and Kulkarni, S. R., "Precision Astrometry With Adaptive Optics," AJ 137, 83-93 (Jan. 2009).

[10] Lu, J. R., Orbits and Origins of Young Stars at the Galactic Center, PhD thesis, UCLA (June 2008).

[11] Clarkson, W., Lu, J. R., , Ghez, A., Morris, M., McCrady, N., Stolte, A., and Yelda, S., "A sharper look at the motion of stars in the Arches with Keck-LGS adaptive optics.," Proceedings of "The Galactic Center: A Window on the Nuclear Environment of Disk Galaxies" (2010).

[12] Teoste, R., Daley, Jr., J. A., Capes, Jr., R. N., Alves, J. J., and Zimmerman, M. D., [Measurements of tilt anisoplanatism at the Firepond Facility], Unknown (Nov. 1988).

[13] Gubler, J. and Tytler, D., "Differential Atmospheric Refraction and Limitations on the Relative Astrometric Accuracy of Large Telescopes," PASP 110, 738-746 (June 1998).

[14] Fritz, T., Gillessen, S., Trippe, S., Ott, T., Bartko, H., Pfuhl, O., Dodds-Eden, K., Davies, R., Eisenhauer, F., and Genzel, R., "What is limiting near-infrared astrometry in the Galactic Centre?," MNRAS 401, 1177-1188 (Jan. 2010).

[15] Diolaiti, E., Bendinelli, O., Bonaccini, D., Close, L., Currie, D., and Parmeggiani, G., "Analysis of isoplanatic high resolution stellar fields by the StarFinder code," AESAS 147, 335-346 (Dec. 2000).

[16] Hornstein, S. D., Matthews, K., Ghez, A. M., Lu, J. R., Morris, M., Becklin, E. E., Rafelski, M., and Baganoff, F. K., "A Constant Spectral Index for Sagittarius A* during Infrared/X-Ray Intensity Variations," ApJ 667, 900-910 (Oct. 2007).

[17] Eckart, A., Baganoff, F. K., Zamaninasab, M., Morris, M. R., Schödel, R., Meyer, L., Muzic, K., Bautz, M. W., Brandt, W. N., Garmire, G. P., Ricker, G. R., Kunneriath, D., Straubmeier, C., Duschl, W., Dovciak, M., Karas, V., Markoff, S., Najarro, F., Mauerhan, J., Moultaka, J., and Zensus, A., "Polarized NIR and X-ray flares from Sagittarius A*," A\&\&A 479, 625-639 (Mar. 2008). 
[18] Do, T., Ghez, A. M., Morris, M. R., Yelda, S., Meyer, L., Lu, J. R., Hornstein, S. D., and Matthews, K., "A Near-Infrared Variability Study of the Galactic Black Hole: A Red Noise Source with NO Detected Periodicity," ApJ 691, 1021-1034 (Feb. 2009).

[19] Harayama, Y., Eisenhauer, F., and Martins, F., "The Initial Mass Function of the Massive Star-forming Region NGC 3603 from Near-Infrared Adaptive Optics Observations," ApJ 675, 1319-1342 (Mar. 2008).

[20] Do, T., Physical Processes in the Vicinity of a Supermassive Black Hole, PhD thesis, UCLA (Aug. 2010).

[21] Schödel, R., "Accurate photometry with adaptive optics in the presence of anisoplanatic effects with a sparsely sampled PSF. The Galactic center as an example of a challenging target for accurate AO photometry," AछA 509, A58+ (Jan. 2010).

[22] Esslinger, O. and Edmunds, M. G., "Photometry with adaptive optics: A first guide to expected performance," A\&AS 129, 617-635 (May 1998).

[23] Stolte, A., Morris, M., Ghez, A., Do, T., Lu, J., Wright, S., Ballard, C., Mills, E., and Matthews, K., "Disks in the Arches cluster - survival in a starburst environment," ArXiv e-prints (June 2010).

[24] Sheehy, C. D., McCrady, N., and Graham, J. R., "Constraining the Adaptive Optics Point-Spread Function in Crowded Fields: Measuring Photometric Aperture Corrections," ApJ 647, 1517-1530 (Aug. 2006).

[25] Veran, J., Rigaut, F., Maitre, H., and Rouan, D., "Estimation of the adaptive optics long-exposure pointspread function using control loop data.," Journal of the Optical Society of America A 14, 3057-3069 (Nov. 1997).

[26] Britton, M. C., "The Anisoplanatic Point-Spread Function in Adaptive Optics," PASP 118, 885-900 (June 2006). 